Cahiers $d u$ MONDE RUSSE

\section{Cahiers du monde russe}

Russie - Empire russe - Union soviétique et États indépendants

$48 / 4 \mid 2007$

Varia

\title{
Petr Vasil'evič Vologodskij, Vo vlasti i v izgnanii
}

Sabine Breuillard

\section{OpenEdition \\ Journals}

Édition électronique

URL : https://journals.openedition.org/monderusse/6072

DOI : 10.4000/monderusse.6072

ISSN : $1777-5388$

Éditeur

Éditions de l'EHESS

Édition imprimée

Date de publication : 2 décembre 2007

Pagination : 694-700

ISBN : 978-2-7132-2148-4

ISSN : $1252-6576$

\section{Référence électronique}

Sabine Breuillard, "Petr Vasil'evič Vologodskij, Vo vlasti i v izgnanii », Cahiers du monde russe [En ligne], 48/4 | 2007, mis en ligne le 28 décembre 2009, consulté le 03 septembre 2022. URL : http:// journals.openedition.org/monderusse/6072 ; DOI : https://doi.org/10.4000/monderusse.6072

Ce document a été généré automatiquement le 3 septembre 2022.

Tous droits réservés 


\title{
Petr Vasil'evič Vologodskij, Vo vlasti i v izgnanii
}

\author{
Sabine Breuillard
}

\section{RÉFÉRENCE}

Petr Vasil'evič VologodsKIJ, Vo vlasti i v izgnanii. Dnevnik prem'er-ministra antibol `̌sevitskih pravitel'stv i emigranta v Kitae (1918-1925) | In Power and in Exile. The Diary of a Prime Minister of anti-Bolshevik Governments and of an Émigré in China (1918-1925). N. S. Lar'kov, S. M. Liandres, D. G. Wulff, éds.Riazan: P. A. Tribunskij, 2006, 619 p. (Novejšaja rossijskaja istorija : issledovanija i dokumenty, 9)

1 Saluons la très belle publication, minutieuse et précise, du Journal que tint pendant sept ans (1918-1925) Petr Vasil'evič Vologodskij, premier ministre dans trois gouvernements antibolcheviks, en particulier le dernier, celui de l'amiral Kolčak, appelé Gouvernement d'Omsk ; il y avait été désigné président du Conseil à la tête duquel il passa dix-sept mois d'une intense activité.

2 Sibérien d'origine, juriste de formation, Vologodskij (1863-1925) naît dans la famille d'un pope de la province de l'Ienisseï. Formé au lycée de Tomsk, puis à la faculté de droit de Saint-Pétersbourg dont il est assez vite exclu pour ses activités politiques, il termine ses études à l'université de Kharkov. Revenu en Sibérie, il prend des fonctions de juge et se lance en politique. Élu député à la deuxième Douma en 1907, il se fait l'ardent défenseur des intérêts régionaux de la Sibérie. Le Gouvernement Provisoire le nomme en 1917 président du palais de justice d'Omsk. La chute de l'Ancien Régime fait naître en Sibérie, territoire traditionnellement considéré comme "colonie " et "terre à convicts", l'espoir d'une autonomie régionale, sans parler pour autant de séparatisme. Mais les opinions sont trop contrastées en 1917 entre socialistes-révolutionnaires, cadets et mencheviks pour parvenir à un consensus. Les bolcheviks arrivés au pouvoir, avec leur tendance à tout centraliser, vont mettre fin à ces rêves d'autonomie. Leur rejet de la tradition juridique fait de Vologodskij, juriste et régionaliste, un opposant résolu. Il 
accueille avec enthousiasme les premiers succès de la légion tchécoslovaque sur le Transsibérien et se réjouit à l'annonce de la fuite des bolcheviks qui abandonnèrent Novonikolaevsk et Tomsk fin mai 1918.

3 Convaincu que la révolution prend un tour nouveau, il décide de tenir son journal. L'espoir vient de naître qu'une restauration d'un État russe uni et démocratique peut se faire à partir de la Sibérie. La conviction qu'il vit des moments très importants de l'histoire de son pays le conduit à rédiger cette chronique, et lui en dicte d'emblée le ton et le style. Très peu d'annotations personnelles, sauf sur son état de santé qui s'aggrave de jour en jour au fil des ans. Très peu aussi sur sa famille, sauf plus tard, quand elles renseignent sur la situation de l'émigré. Le lecteur n'a pas accès direct à son monde intérieur.

4 Vologodskij se permet rarement de médire - pratique courante dans les Mémoires - de tel ou tel personnage dont on sait par ailleurs qu'il ne l'appréciait guère. Et s'il relate quelque acte condamnable, c'est lorsque l'auteur l'a blessé dans son amour-propre en critiquant d'une manière injuste son œuvre de premier ministre. Mais il supporte le reproche, admet la critique et, sine ire et studio, l'inscrit dans son cahier, fidèle au style factuel du chroniqueur.

5 Il y a à cela deux raisons : le projet d'écrire plus tard ses Mémoires (les carnets lui serviront de matrice) et le souci de garder une trace de ces événements politiques et historiques, même sous la forme d'un journal personnel, souvent rédigé en style télégraphique au cours de ses nombreux déplacements en tant que ministre. Qui sait « si quelque catastrophe ne viendra pas détruire ces protocoles du conseil des ministres », écrit-il, lucide, en relatant son action, habité par la hantise du grand anéantissement, de l'effacement de tout témoignage, de l'oubli de ce nouveau Temps des Troubles de la Russie. La chronique quasi journalière des événements de la guerre civile en Sibérie, dont il est un acteur important, puis de sa vie d'émigré en Chine, doit selon lui, grâce aux informations de première main qu'il note, pallier les risques de destruction de documents liés aux aléas de la révolution autant que les insuffisances de la presse locale ou étrangère trop souvent soumises à la désinformation bolchevique.

6 C'est là son projet original. Vologodskij publie à Pékin en 1920, dans une revue de l'émigration russe Russkoe obozrenie, les trois premiers chapitres de ses Mémoires sur son enfance, sa jeunesse et sa vie d'étudiant. Il a sans doute l'intention de continuer sur son action politique, à partir des notes du Journal. Mais la vie en décide autrement. Le 24 septembre 1924, l'administration de Chinese Eastern Railway (CER, Chemin de fer de la Chine de l'Est, l'Est chinois) passe aux mains des Soviétiques et des Chinois. Il est licencié quatre mois après. Pour garder son poste de juriste auprès de l'Est chinois, il aurait dû adopter la nationalité soviétique ; c'était aller contre ses convictions profondes. Il tente d'adopter la nationalité chinoise, s'y prend trop tard: il était déjà remplacé par un Chinois! Privé de son unique gagne-pain, n'étant plus en mesure de nourrir sa famille, il sombre dans une profonde dépression et meurt à Harbin le 19 octobre 1925.

7 Il œuvra jusqu'au bout, apportant les dernières corrections d'un texte qui l'accompagna pendant toutes ces années. Il avait même en 1924 mis au net une partie de son Journal (6 juin 1918-21 mars 1920) en vue d'une publication dans la collection Archives de la révolution russe que dirigeait I.V. Gessen à Berlin. Le manuscrit ne fut pas publié. Ces pages ont été retrouvées au GARF (Archives nationales de la Fédération de Russie) dans les Archives de Prague, archives de l'émigration russe, « offertes » comme on sait à la fin de la guerre «au vainqueur en signe de reconnaissance », puis déménagéesà Moscou. 
Elles furent publiées après la perestroïka, à la va-vite, sans appareil critique, dans le recueil Rossija antibol'ševitskaja. Iz belogvardejskih i emigrantskih arhivov [La Russie antibolchevique. Extrait des archives de l'émigration blanche] en 1995.

Dès 1933, sa veuve tenta de vendre à la Hoover Institution les sept cahiers du Journal, avec l'aide pourtant du juriste Gins, alors encore à Harbin. Ce fut sans succès. Il a fallu attendre 2007 pour que le texte intégral voie le jour.

9 La présente édition est publiée dans les règles de l'art. Tout concourt à en faire un ouvrage solide à l'usage des historiens. C'est le fruit d'une étroite collaboration entre la fille de l'auteur, Zinaida Petrovskaja Popova - qui réside aujourd'hui en Australie, comme tant d'autres anciens Russes de Harbin - et le groupe de rédacteurs qui ont initié à Riazan cette très intéressante nouvelle série : Novejšaja rossijskaja istorija : issledovanija $i$ dokumenty (Histoire russe moderne et contemporaine: monographies et documents d'archives). Le comité de rédaction du livre et de la série est international; on y reconnaît les noms d'éminents historiens de la Russie.

L'ouvrage s'ouvre sur une bonne introduction qui restitue l'histoire du Journal et la personnalité de Vologodskij. Le corps (les sept années du Journal) est suivi d'un appareil de notes solidement documentées, puisées tant dans les archives que dans l'ample bibliographie finale. Cette dernière constitue à elle seule une bonne initiation pour quiconque s'intéresse au mouvement blanc, à l'histoire de l'émigration russe, et plus particulièrement à l'émigration russe en Chine.

11 Un index des principaux noms propres cités, annotés autant que faire se peut, où se dégagent quelques grands noms du mouvement antibolchevik de Sibérie; un index des noms géographiques, quelques photos d'époque de la ville de Tomsk et de la famille Vologodskij, une carte des années 1920 de la Russie asiatique, une liste enfin des sigles complètent ce précieux appareil critique et font de ce livre un outil incontournable pour tout historien de la période.

Il serait trop long d'entrer dans le détail de chaque année, et il n'est pas question de les résumer non plus. Laissons au lecteur le soin de pénétrer dans le tissu même de la révolution et de l'exil, et limitons-nous ici à quelques impressions globales qui s'efforceront de traduire le ton d'un homme et d'une époque.

La valeur de ce Journal tient essentiellement au caractère factuel des événements relatés et aux personnages mis en scène impliqués dans le mouvement antibolchevik en Sibérie. On y trouvera des informations très précises sur le travail des ministres et la politique mise en œuvre et sur les initiatives plus ou moins heureuses, voire arbitraires, des généraux blancs et de leurs officiers souvent ingouvernables. À propos de ces derniers, Vologodskij rapporte ce que Kolčak lui dit un jour : « Je n'arrive pas moi-même à diriger l'ataman Semenov, comment pourrais-je exiger de Stepanov qu'il vienne à bout de ce phénomène pratiquement incontrôlable de la débandade des officiers qui versent dans le hooliganisme».

14 Cela est surtout valable pour les deux premiers cahiers (1918 et 1919) jusqu'à la chute du Gouvernement d'Omsk. Chaque événement est décrit, parfois annoté succinctement. L'homme politique se permet rarement quelque commentaire. Il le déplorera en 1924 quand il se relira, estimant que, dans le feu de l'action, il n'avait pas donné assez d'interprétations et de jugements politiques sur les événements dont il était le témoin. Mais cette vision factuelle des choses, sans recul, n'est-elle pas le propre de tout témoin au milieu de la mêlée, atteint d'une forme de myopie tel Fabrice del Dongo à Waterloo ? 
Si intervention personnelle il y a, elle serait davantage à rechercher du côté du montage des événements, de leur présentation, qui serait en quelque sorte une réinterprétation du réel. Et peut-être même que sans montage aucun, il n'y a qu'une simple restitution d'une réalité qui se déroulait sous ses yeux et lui résonnait aux oreilles quotidiennement.

Cela dit, sa peinture très juste des différents acteurs permet de mieux suivre les fluctuations du mouvement blanc, d'en comprendre les erreurs, les raisons enfin de l'échec du gouvernement de Kolčak. Or ces erreurs, Vologodskij, en tant que président du conseil du Gouvernement d'Omsk, en portait aussi la responsabilité. Il en était conscient. Rappelons néanmoins qu'il se retrouva souvent bien seul face à des ministres qui démissionnaient les uns après les autres et fuyaient.

Cet homme de conviction et d'engagement n'a pas toujours eu un sens politique éclairé. Un environnement difficile, où les nouvelles les plus contradictoires se pressaient, ne favorisait pas une appréciation exacte de la situation. Il n'a pas su prévoir avec la sagacité suffisante le retour des bolcheviks en Sibérie. Convaincu que « le bolchevisme tomberait de soi-même comme était tombé le tsarisme, comme les Allemands s'étaient rendus ", il appelait les Japonais et les Tchèques à aider la Sibérie à repousser l'attaque de l'Armée rouge, sûr de regagner ensuite la confiance de la population. Il ne pensait pas que les bolcheviks désiraient pousser jusqu'en Sibérie orientale. Pourtant, le 25 octobre 1919, il faut se rendre à l'évidence de l'avancée des Rouges. Le Gouvernement d'Omsk prend la décision de se replier sur Irkoutsk. C'est par la presse que Vologodskij apprend le 28 octobre « qu'il avait décidé de quitter son poste de président du conseil pour raison de santé » : le gouvernement, tout en déménageant en train à Irkoutsk, avait été remanié.

Début janvier 1920, le gouvernement Kolčak vit ses derniers jours. Vologodskij est arrêté dans la rue, désarmé et relâché. Le 8 janvier, dans un manifeste, Kolčak, au même titre que les atamans Semenov et Kalmykov, est déclaré « ennemi du peuple ».

février, après avoir fui Irkoutsk, Vologodskij a pu gagner Harbin en train grâce à l'aide de la mission militaire japonaise. Il se retrouve dans un même wagon que d'autres membres du Gouvernement d'Omsk qui constitueront bientôt l'intelligentsia de Harbin : G.K. Gins, A.N. Gattenberger, N.V. Ustrialov. Une page est tournée, un nouveau chapitre s'écrit, celui de l'exil en Chine : c'est le sujet des cinq cahiers de 1920 à 1925.

Ce séjour en Chine, les cinq dernières années de sa vie, est intéressant à plus d'un titre. Il nous renseigne sur la difficile existence des émigrés russes que les Chinois, qui avaient connu l'arrogance impériale des colons russes - les "grands capitaines »- envers les coolies au début du siècle, considéraient maintenant comme des Russes déchus (p. 451). Les rôles étaient inversés. Çà et là on prenait sa revanche. Vologodskij nous décrit aussi avec beaucoup de détails intéressants, parfois piquants et pittoresques, avec presque une vision d'exote ${ }^{1}$ la situation politique de la Chine livrée aux luttes pour le pouvoir entre seigneurs de la guerre, en particulier entre Zhang Zuolin en Mandchourie et Wu Peifu à Pékin. Il conte avec beaucoup de verve sa visite dans le vieux quartier mandchou de Pékin chez un général chinois, ancien honghuzy, qui l'avait invité avec d'autres experts russes dont il escomptait l'aide pour exploiter, à son profit, une mine d'or au nord de la capitale chinoise.

Mais, aspect non moins important et souvent mal éclairé de l'historiographie, le lecteur glanera dans ce Journal des informations très précises sur la situation créée à la suite de l'effondrement de la Russie tsariste, ainsi que sur les problèmes diplomatiques qui surgirent alors entre la Chine et la Russie. La chute de l'Empire tsariste sonna le glas du système des concessions étrangères en Chine mis en place à la suite des traités inégaux 
des guerres de l'opium, remettant profondément en cause la propriété et la direction de la concession ferroviaire de l'Est chinois, Kitajskaja Vostočnaja Železnaja Doroga (KVŽD). À Pékin, où Vologodskij séjourna plusieurs mois, nous assistons à la restructuration de la Banque russo-asiatique, au remerciement des diplomates de l'Ancien Régime tsariste, aux intrigues diplomatiques des bolcheviks. Nous suivons la valse hésitation des Puissances à reconnaître l'Union soviétique.

Ces cinq livrets sont dominés par une note nostalgique, le sentiment de la fin d'une époque et d'une extrême impuissance à agir sur l'événement. Vologodskij compare l'effondrement de la Russie à celui de l'Empire romain. L'espoir d'une renaissance d'une Russie grande et unie s'effiloche au fil des pages.

La difficulté de trouver un emploi correspondant à sa formation de juriste le contraint dès son arrivée en Chine à se rendre là où il $\mathrm{y}$ avait une présence russe. C'est Harbin d'abord, enclave russe au cœur de la Mandchourie, seul endroit où il se sentira vraiment chez lui, mais aussi centre administratif de l'Est chinois, ville en pleine expansion commerciale et industrielle, qu'un de ses amis lui décrit comme un nouveau Chicago. C'est Shanghai ensuite, Tianjin, Qingdao, Pékin enfin où se trouvaient la Mission russe et la Banque russoasiatique. Il ne va pas jusqu'à Hankou ${ }^{2}$ sur le Yangtsé, autre concession russe, mais il en parle. Cette première prise de contact le convainc de la « faible influence de la Russie en Chine ». Il se fixera quelque temps à Tianjin pour revenir à Harbin où l'arrivée massive des Russes exilés rendait pourtant encore plus aléatoire la possibilité de trouver un emploi.

À travers ses cahiers, on suit les derniers soubresauts du mouvement blanc, la formation de la République d'Extrême-Orient à Vladivostok, la stratégie des Japonais auxquels une partie des officiers blancs se disaient prêts à céder sur tous les points: Sakhaline, le Kamtchatka, la pêche et l'Est chinois, pourvu qu'ils les aident à chasser les bolcheviks. Il apprend par la presse l'arrestation de Kolčak et de son gouvernement, puis la condamnation à mort de l'amiral et de quatre de ses ministres, les autres étant condamnés aux travaux forcés. Il comprend alors que s'il n'avait pas fui, il aurait été fusillé..., éprouve un sentiment de soulagement d'avoir échappé au massacre, à l'exécution, au déshonneur. Toutefois ces informations, soit orales soit écrites, lui parviennent difficilement et se trouvent parfois démenties trois mois plus tard. Tout ceci accentue son isolement, le sentiment d'être coupé d'un « essentiel » qui se passe ailleurs. Cette impression d'avoir été dépassé, de se retrouver on the shore, ne viendra pas pour autant dynamiser les sursauts d'une action de mobilisation à partir du "Procheétranger ». D'ailleurs l'eût-il voulu, c'eût été pratiquement impossible: l'émigration russe était bien trop divisée.

Il décrit néanmoins la situation à Harbin au début des années 1920 avec la précision de l'entomologiste et l'énergie du témoin: la grève du chemin de fer sur mot d'ordre (téléguidé) des bolcheviks, l'éviction de son directeur, le général Horvat; la reprise en main progressive par les Chinois du Chemin de fer de la Chine de l'Est, encouragés dans cette voie par les bolcheviks qui, tout en dénonçant dans un geste faussement désintéressé les traités inégaux, se disaient prêts à le rétrocéder à la Chine, mais n'en désiraient pas moins secrètement garder la haute main dessus. Le récit sur les négociations de Ioffe est intéressant à ce sujet.

Vologodskij assiste impuissant à cette débâcle russe. Les Chinois prennent en main la gestion de la municipalité de Harbin, remettent en cause, puis abolissent le droit d'extraterritorialité pour les Russes en Chine. Cela allait réduire plus encore les chances du juriste Vologodskij de trouver un travail. En effet, il n'apprendra pas le chinois, se 
sentant trop âgé pour cela. En revanche, il se mettra avec courage et détermination à l'anglais.

Le sentiment d'étrangeté vis-à-vis de son nouvel environnement est de plus en plus prégnant, quels que soient ses efforts pour participer à la structuration sociale de cette société émigrée et ses tentatives pour reconstituer des liens culturels intracommunautaires dans la colonie russe, en particulier à Tianjin. Le mal du pays pour sa Sibérie l'étreint régulièrement. Noël à l'étranger («na čužbine»), l'absence d'horizon, un ciel de plomb, ses économies qui s'engloutissent chaque jour davantage dans les locations d'hôtels garnis ou de meublés, l'espoir de retour en Russie qui s'amenuise jour après jour car les bolcheviks triomphent en dépit des famines régionales, des grèves d'ouvriers, des révoltes paysannes.

Que ce document ait enfin trouvé éditeur n'est que justice. Sa qualité et la précision de ses informations en font un document unique, de première main, sur la guerre civile et les premières années de l'émigration russe en Chine, même si l'on a parfois l'impression que Vologodskij se noie dans le détail : mais n'est-ce pas la loi du genre? Le gagne-pain qui ne vient pas, les petites tractations sordides sur les salaires, les informations sur la gestion de l'Est chinois, les luttes de rivalités, peignent le quotidien sans fard de l'émigré. Il y a aussi cette nostalgie très forte de la russité en terre étrangère, couplée de l'étrange sentiment que le contenu même de la russité a changé, qu'il n'est plus le même qu'avant 1917 tel que les émigrés blancs, avec entêtement, cherchaient encore à le faire perdurer dans un temps figé. Qu'on l'accepte ou pas, la réforme du calendrier russe avait eu lieu en 1917 ! Cette russité avait désormais des résonances nouvelles encore difficiles à saisir qui se heurtaient à celles d'une Chine qui elle-même changeait. Tout cela est parfaitement rendu.

Ce Journal nous entraîne au cœur d'une histoire sur laquelle commencent à paraître quelques travaux. Il vient compléter heureusement les journaux et mémoires des acteurs de la guerre civile publiés dans l'entre-deux-guerres. Sans parler du recueil de G.K. Gins, Sibir', sojuzniki i Kolčak [La Sibérie, les Alliés et Kolčak] paru à Pékin en $1921^{3}$ et devenu un classique, nous pensons en particulier aux Mémoires du socialiste-révolutionnaire M.A. Krol'4 qui émigra en Chine en 1918 et gagna ensuite la France où il mourut en 1942, et à ceux d'I.I. Serebrennikov ${ }^{5}$, ministre des Affaires étrangères du Gouvernement d'Omsk, mort en 1953 à Tianjin. Et pour compléter le chapitre de la présence russe en Extrême-Orient à cette époque, citons les publications, l'une récente, l'autre prochaine, concernant deux diplomates russes : les Mémoiresdu fils du dernier consul général ${ }^{6}$ de la Russie impériale à Moukden et ceux de D.I. Abrikossov à Tokyo.

\section{NOTES}

1. Au sens de Victor Segalen, voir : Essai sur l'exotisme : une esthétique du divers, P. : Fata Morgana, 1978.

2. Actuel Wuhan. 
3. G.K. Gins, Sibir', sojuzniki i Kolčak. Povtornyj moment russkoj istorii, 1918-1920gg. (Vpečatlenija i mysli člena Omskogo pravitel'stva), t. I-II, Pékin : Tipo-lit. Russkoj-duhovnoj missii, 1921.

4. M.A. Krol', Za tri goda (Vospominanija, vpečatleniija i vstreči), Vladivostok : Biblioteka svobodnoj Rossii, $\mathrm{n}^{\circ} 17,1921$.

5. I.I. Serebrennikov avait publié ses Mémoires à Tianjin en 1937 et $1940:$ Moi vospominanija, en 2 t.,t. 1 : V Revoliucii (1917-1919) ; t. 2 : V emigracii (1920-1940), Tianjin :Tip. «Star Press », 1937 et Naše znanie, 1940. Son Journal en cinq volumes constituera le tome 8 de cette même série Novejšaja rossijskaja istorija : issledovanija i dokumenty, publié cette fois avec l'aide de la Hoover Institution et des éditions ROSSPEN : Kitaj i russkaja emigracija $v$ dnevnikah I. I. i A.N. Serebrennikovyh. Le tome 1 est paru en 2006, sous la direction d'Oleg Budnickij et de Terence Emmons, avec une introduction et des notes d'A.A. Hisamutdinov.

6. M.Ju. Sorokinoj, E.B. Kuznecovoj, B.S. Kolokolov, éds., Zapiski syna poslednego rossijskogo imperatorskogo general'nogo konsula $v$ Mukdene, SPb. : Athenaeum-Feniks, 2007, p. 3-68 (Diaspora : Novye materialy, 9).

7. Abrikossov a d'abord été publié en anglais: G.A. Lensen, éd., D.I. Abrikossow, Revelations of a Russian Diplomat: The Memoirs of Dmitrii I. Abrikossow, Seattle, WA: University of Washington Press, 1964. Le texte complet paraîtra l'été prochain en russe. Actuellement, sur le Japon, seule une partie a été publiée: M.Ju. Sorokinoj, éd., «Dmitrij Abrikosov. Tokio: posol'stvo bez gosudarstva ", Meždunarodnaja žizn', 4, 2007, p. 100-132. 